\title{
Physically based diagonal treatment of polar optical phonon self-energy: performance assessment of III-V double-gate transistors
}

\author{
Manel Moussavou, ${ }^{1}$ Michel Lannoo, ${ }^{1, *}$ Nicolas Cavassilas, ${ }^{1}$ Demetrio Logoteta,${ }^{1}$ and Marc Bescond ${ }^{2, \dagger}$ \\ ${ }^{1}$ IM2NP, UMR CNRS 7334, Aix-Marseille Université, Technopôle de Château-Gombert, \\ Bâtiment Néel, 60 Rue Frédéric Joliot Curie, 13453 Marseille, France \\ ${ }^{2}$ LIMMS, CNRS-UMI 2820, Institute of Industrial Science, University of Tokyo, 153-8505 Tokyo, Japan
}

\begin{abstract}
We propose a diagonal approximation for the self-energy that describes the interaction between electrons and polar optical phonons in the framework of non-equilibrium Green's function transport simulations. Our model is based on the definition of a scaling factor, which renormalizes the local electron-phonon coupling, to take into account the non-locality of the interaction and provide the correct scattering rates. While previous studies relied on empirical values of this factor, we derive, from basic physical relationships, analytical expressions in the presence of one- and two-dimensional confinement of phonons. We apply our model to the self-consistent simulation of double-gate p-type transistors made of technologically relevant III-V materials (InAs, InSb and GaSb). Their performance are benchmarked, for different crystallographic orientations and strain constraints, against the corresponding Si and Ge devices. We find that the electron-polar optical phonon scattering plays a major role in degrading the performance of the III-V devices and typically results in a widening of the performance gap existing between III-V and Si or Ge devices in ballistic transport conditions.
\end{abstract}

\section{INTRODUCTION}

During more than 40 years the impressive growth of semiconductor industry was based on the scaling of the transistor, which represents the elementary brick of integrated circuits. However, since the $90 \mathrm{~nm}$ node, the device scaling in itself is no more capable to maintain this continuous technological development. Additional technological boosters (e.g, high- $\kappa$ dielectric and mechanical strain) were first incorporated to improve the electrical characteristics of transistors [1]. Multiple gate Metal Oxide Semiconductor Field-Effect-Transistors (MOSFETs) have also been developped to counteract the short channel effects when reaching the nanometer scale $[2,3]$.

Another important option is to consider alternative channel materials with better electrical properties than Si $[4-6]$. Due to their very high electron mobility, III-V materials have been recognized to be good channel material candidates $[7,8]$. While several technological [9] and physical [10] challenges must be overcome to incorporate III-V devices in future CMOSFETs, they are considered as a promising solution to meet the power constraints arising in several applications. The recent International Roadmap for Devices and Systems (IRDS) pointed out the achievement of high performances p-type strained III$\mathrm{V}$ MOSFETs as key near-term challenges to address for the device scaling [11].

A drawback of using III-V materials for a monolithic integration is the large imbalance between electron and hole mobilities (e.g., in bulk InAs, the electron mobility is $\mu_{e}=40 \times 10^{3} \mathrm{~cm}^{2} \cdot \mathrm{V}^{-1} \cdot \mathrm{s}^{-1}$ while the hole mobility is $\mu_{h}=500 \mathrm{~cm}^{2} \cdot \mathrm{V}^{-1} \cdot \mathrm{s}^{-1}$ ) [12]. Since CMOS circuits require both $\mathrm{n}$ - and p-channels with reasonably matched performances, the future III-V CMOS technology is required to narrow the gap between n- and p-type MOSFETs.
The use of mechanical strain represents a relevant technique to reach this aim [13-16]. Therefore, comprehensive investigations on III-V pMOSFETs are urgently needed in order to better predict and optimize their performances with respect to the choice of the material, mechanical strain and crystallographic orientation.

Quantum transport modeling can play a central role to determine the best device configuration. Most theoretical studies have so far explored the n-type III-V MOSFETs [17-20] while few investigations focused on their p-type counterparts. Indeed, modeling hole transport in semiconductor nanostructures is typically a more complex task, as it requires to take into account the coupling between several bands $[21,22]$. The situation is even more complicated when strain and phonon scattering are included [23-25].

Non-Equilibrium Green's Function (NEGF) formalism is one of the most established approach to treat quantum transport in nano-devices [26-28]. It is able to describe inelastic scattering, like electron-phonon interactions, through the concept of self-energy [29]. For numerical convenience the electron-phonon scattering is usually treated within a local approximation, since most of the NEGF codes are based on a recursive algorithm [30, 31], which requires a diagonal (i.e. local) description of the self-energy. This assumption was demonstrated to be physically sound for interactions with acoustic and nonpolar optical phonons [32-35].

However, III-V compound semiconductors are subject to strong polar optical (PO) interactions of the Fröhlich type [36-38], which intrinsically have a non-local character. Indeed, this type of electron-phonon interaction decays very slowly in space like a Coulomb potential [36]. This non-locality increases the numerical complexity of the quantum transport computations in a prohibitive manner. Previous NEGF studies have proposed to model 
PO phonon scattering through diagonal self-energies multiplied by a scaling factor [39], but without providing general prescriptions.

In this paper, by directly starting from the Fröhlich theory of bulk polar optical phonons, we derive an analytical expression of the scaling factor in confined systems, which can be used to mimic the effects of polar optical phonons within a local approximation. We then use our model to calibrate the electron-PO phonon coupling strength in self-consistent NEGF simulations, in order to assess the performances of p-type double-gate (DG) MOSFETs as a function of the channel material, of the crystallographic orientation and of the strain.

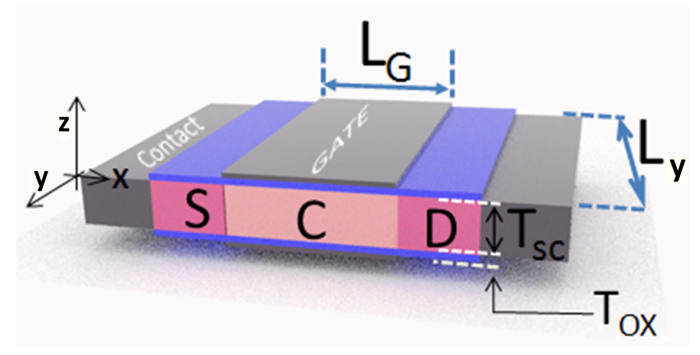

FIG. 1. Sketch of the considered Double-Gate MOSFETs. For all the considered devices, $T_{S C}=2.4 \mathrm{~nm}, T_{O X}=1 \mathrm{~nm}$, and the source and drain doping is $8 \times 10^{19} \mathrm{~cm}^{-3}$. Two gate lengths, $L_{G}=15 \mathrm{~nm}$ and $7 \mathrm{~nm}$, are considered. $V_{D S}=-0.6 \mathrm{~V}$.

We use a two-dimensional (2D) NEGF code selfconsistently coupled with the Poisson equation [40, 41]. The valence bandstructure of III-V direct gap materials is described by a 8-band k.p Hamiltonian [42].

The paper is organized as follows: Section presents the NEGF framework. In Section, an expression of the PO phonon self-energy is derived, first in the bulk case and then for a thin film DG MOSFET. In Section, the diagonal approximation is introduced, and an analytic expression of the scaling factor is derived. In Section , we present and discuss the results of our numerical simulations of III-V DG MOSFETs. Section IV summarizes the key findings.

\section{METHOD}

Quantum kinetic equation within the eight-band $k \cdot p$ framework

We consider a 8 -band $k \cdot p$ Hamiltonian including the spin-orbit coupling to model the valence bandstructure of the 2D DG pMOSFET shown in Fig. 1. The DG pMOSFET geometry is sketched in Fig. 1. We model the valence band structure through a 8 -band $k \cdot p$ Hamiltonian, which is able to take into account the coupling between the light, heavy and split-off hole bands, and the coupling between them and the conduction band [42, 43]. The device is assumed translationally invariant in the transverse y direction. Accordingly, the Hamiltonian can be parametrized in terms of the wave vector component $k_{y}$. In order to obtain accurate results, in numerical computations, a large enough number of suitably spaced values of $k_{y}$ has to be considered. We chose $k_{y}$ of the form $2 \pi n / L_{y}$, with $\mathrm{n}$ an integer and $L_{y}=10 \mathrm{~nm}$.

Mechanical strain is modeled through the Bir-Pikus Hamiltonian, and by assuming bulk values of deformation potentials [44]. The bandstructure material parameters are extracted from reference 45 and reported in Table I.

TABLE I. Principal $k \cdot p$ parameters used in this work [45].

\begin{tabular}{lccccccc}
\hline \hline & $\gamma_{1}^{L}$ & $\gamma_{2}^{L}$ & $\gamma_{3}^{L}$ & $E_{g}$ & $E_{p}$ & $\Delta_{\text {so }}$ & $a_{0}$ \\
\hline InAs & 20.0 & 8.5 & 9.2 & 0.417 & 21.5 & 0.39 & 6.06 \\
InSb & 34.8 & 15.5 & 16.5 & 0.23 & 23.3 & 0.81 & 6.48 \\
GaSb & 13.4 & 4.7 & 6.0 & 0.81 & 27 & 0.76 & 6.09 \\
Units & & & $\mathrm{eV}$ & $\mathrm{eV}$ & $\mathrm{eV}$ & $\AA$ \\
\hline \hline
\end{tabular}

To solve the transport problem in the presence of inelastic interactions, the Keldysh retarded and lesser/greater-than Green's functions are calculated for each transverse mode $k_{y}$ by solving the following equations (in matrix notation):

$$
\begin{aligned}
{\left[G^{R}\left(k_{y}, \epsilon\right)\right]=\left\{\epsilon[I]-\left[H^{2 D}\left(k_{y}\right)\right]-[U]\right.} \\
\left.-\left[\Sigma_{L}^{R}\left(k_{y}, \epsilon\right)\right]-\left[\Sigma_{\text {scat }}^{R}\left(k_{y}, \epsilon\right)\right]\right\}^{-1} \\
{\left[G^{\lessgtr}\left(k_{y}, \epsilon\right)\right]=\left[G^{R}\left(k_{y}, \epsilon\right)\right]\left(\left[\Sigma_{L}^{\lessgtr}\left(k_{y}, \epsilon\right)\right]\right.} \\
\left.+\left[\Sigma_{\text {scat }}^{\lessgtr}\left(k_{y}, \epsilon\right)\right]\right)\left[G^{R \dagger}\left(k_{y}, \epsilon\right)\right],
\end{aligned}
$$

where $\epsilon$ is the hole energy, $I$ is the identity matrix, $\left[H^{2 D}\left(k_{y}\right)\right]$ the 2D Hamiltonian for a given transverse mode and $[U]$ the $2 \mathrm{D}$ potential (in terms of hole energy). To ensure accurate calculations, 10 discrete values of $k_{y}$ are considered in the simulations. In Eqs.(1) and $(2),\left[\Sigma_{L}^{R, \lessgtr}\right]$ are the retarded and lesser/greater-than selfenergies of the leads, respectively. Moreover, $\left[\Sigma_{\text {scatt }}^{R, \lessgtr}\right]=$ $\left[\Sigma_{\mathrm{ac}}^{R, \lessgtr}\right]+\left[\Sigma_{\mathrm{op}}^{R, \lessgtr}\right]+\left[\Sigma_{\text {pop }}^{R, \lessgtr}\right]$, where $\left[\Sigma_{\mathrm{ac}}^{R, \lessgtr}\right],\left[\Sigma_{\mathrm{op}}^{R, \lessgtr}\right],\left[\Sigma_{\mathrm{pop}}^{R, \lessgtr}\right]$ are the acoustic, optical and PO phonon self-energies, respectively. The self-energies of the leads are calculated exactly [46], while the scattering ones are treated within a perturbative approach, through the self-consistent Born approximation (SCBA) [47]. Within the SCBA and by considering the elastic approximation, the hole-acoustic phonon self-energies are expressed as

$$
\begin{aligned}
{\left[\Sigma_{\mathrm{ac}}^{R, \lessgtr}\left(k_{y}, \epsilon\right)\right]=} & i \operatorname{Im}\left\{\sum_{k_{y}} \sum_{\xi \in\{x, y, z\}}\left[M_{\mathrm{ac}, \xi}\right]\right. \\
& {\left.\left[G^{R, \lessgtr}\left(k_{y}, \epsilon\right)\right]\left[M_{\mathrm{ac}, \xi}^{\dagger}\right]\right\}, }
\end{aligned}
$$


where $\left[M_{\mathrm{ac}, \xi}\right]$ describes the hole-acoustic-phonon coupling. The optical phonon lesser/greater-than and retarded self-energies read [48]

$$
\begin{aligned}
{\left[\Sigma_{\mathrm{op}}^{\lessgtr}\left(k_{y}, \epsilon\right)\right] } & =i \operatorname{Im}\left\{\sum_{\xi \in\{x, y, z\}}\left[M_{\mathrm{op}, \xi}\right]\right. \\
& \times\left(N_{\mathrm{op}}\left[G^{\lessgtr}\left(k_{y}, \epsilon-\hbar \omega_{o p}\right)\right]\right. \\
& \left.+\left(N_{\mathrm{op}}+1\right)\left[G^{\lessgtr}\left(k_{y}, \epsilon+\hbar \omega_{o p}\right)\right]\right) \\
& \left.\times\left[M_{\mathrm{op}, \xi}^{\dagger}\right]\right\} \\
{\left[\Sigma_{\mathrm{op}}^{R}\left(k_{y}, \epsilon\right)\right]=} & i \operatorname{Im}\left\{\sum _ { \xi \in \{ x , y , z \} } [ M _ { \mathrm { op } , \xi } ] \left(N_{\mathrm{op}}\left[G^{R}\left(k_{y}, \epsilon-\hbar \omega_{o p}\right)\right]\right.\right. \\
+ & \left(N_{\mathrm{op}}+1\right)\left[G^{R}\left(k_{y}, \epsilon+\hbar \omega_{\mathrm{op}}\right)\right] \\
+ & \frac{1}{2}\left(\left[G^{>}\left(k_{y}, \epsilon-\hbar \omega_{o p}\right)\right]\right. \\
& \left.\left.\left.-\left[G^{>}\left(k_{y}, \epsilon+\hbar \omega_{o p}\right)\right]\right)\right)\left[M_{\mathrm{op}, \xi}^{\dagger}\right]\right\},
\end{aligned}
$$

where $\hbar \omega_{o p}$ is the optical-phonon energy, $N_{\text {op }}=$ $1 /\left(\exp \left(\hbar \omega_{o p} / k_{B} T\right)-1\right)$ is the corresponding phonon distribution, $\left[M_{\mathrm{op}, \xi}\right]$ represents the hole-optical-phonon coupling and a dispersionless approximation has been considered.

We solve Eqs. (1) and (2) through the recursive algorithm described in Refs. 49 and 50. This approach allows one to efficiently compute the diagonal and first off-diagonal blocks of the Green's functions, from which the charge and current densities are obtained. This considerably lightens the computational burden with respect to a direct numerical implementation of Eq. (1), but requires to resort a local approximation for the scattering self-energies. If this approximation is applied to PO phonons, the electron-phonon coupling needs to be renormalized, in order to avoid an underestimation of the scattering rates. In the next Section, we will define a diagonal approximation that locally conserves the electron-PO phonon scattering rates, and derive easy-to-handle analytical expressions for the corresponding scaling factor.

\section{Polar optical phonon self-energy}

We start from the second order expression of the lesser/greater self-energy of the electron-phonon interaction [28]:

$$
\begin{aligned}
{\left[\Sigma^{\lessgtr}(\epsilon)\right] } & =\sum_{\mathbf{q}} \frac{\hbar}{2 \mu \omega} \frac{\partial H_{e p}}{\partial u_{\mathbf{q}}} \\
& \times\left\{\left(N_{p o p}+1\right) G^{\lessgtr}(\epsilon \pm \hbar \omega)+N_{p o p} G^{\lessgtr}(\epsilon \mp \hbar \omega)\right\} \\
& \times \frac{\partial H_{e p}^{\dagger}}{\partial u_{\mathbf{q}}}
\end{aligned}
$$

In Eq. (6), $H_{e p}$ is the electron-phonon interaction Hamiltonian, $\mathbf{q}$ denotes the phonon wave vector, and $u_{\mathbf{q}}$ and $\mu$ are the amplitude of the normal mode relative displacement with respect to the equilibrium position and the reduced mass of the atoms in the unit cell, respectively.

We evaluate Eq. (6) by calculating $H_{e p}=e \phi$, where $e$ represents the elementary charge and $\phi$ the energy due to polar optical phonons. We first consider the bulk situation (Subsection ), and then we derive the case of a quantum well (Section).

\section{Bulk formulation}

In this Subsection, we outline the calculation of the bulk electron-PO phonon interaction, by following the Fröhlich approach [36]. We consider ionic crystals with two atoms per unit cell. In the long wavelength limit, $\mathbf{u}_{\mathbf{q}}(\mathbf{R})$ expresses the relative displacement of the two atoms in the unit cell at $\mathbf{R}$. The associated ionic polarization $\mathbf{P}_{\mathbf{i}}(\mathbf{R})$, defined as the average dipole moment per unit volume, can be written to the first order in the displacement as

$$
\mathbf{P}_{\mathbf{i}}(\mathbf{R})=\frac{e^{*}}{\Omega} \mathbf{u}_{\mathbf{q}}(\mathbf{R})
$$

where $e^{*}$ is the effective charge and $\Omega$ the volume of the unit cell. We assume that only longitudinal PO phonons couple with electrons. In this hypothesis,

$$
\mathbf{u}_{\mathbf{q}}(\mathbf{R})=u_{\mathbf{q}} \frac{\mathbf{q}}{q} \frac{e^{i \mathbf{q} \cdot \mathbf{R}}}{\sqrt{V}}
$$

where, in the long wavelength limit $\mathbf{q} \rightarrow 0$, the displacement can be considered as a continuous function of $\mathbf{R}$ normalized over the volume $V$ of the system. As $\operatorname{div}(\varepsilon \mathbf{E})=\operatorname{div}\left(\mathbf{E}+4 \pi \mathbf{P}_{\mathbf{i}}\right)=i \mathbf{q}\left(\mathbf{E}_{\mathbf{q}}+4 \pi \mathbf{P}_{\mathbf{i q}}\right)=0, u_{\mathbf{q}}$ induces an electrostatic potential $\phi_{\mathbf{q}}$ corresponding to an electric field $\mathbf{E}_{\mathbf{q}}=-4 \pi \mathbf{P}_{\mathbf{i q}}=-i \mathbf{q} \phi_{\mathbf{q}}$ (in the electrostatic esu-cgs unit system):

$$
\phi_{\mathbf{q}}=-\frac{i}{q} \frac{4 \pi e^{*}}{\Omega} u_{\mathbf{q}}
$$

One still has to determine $e^{*}$. For this, we first notice that a finite static $\mathbf{u}_{\mathbf{q}}$ can be induced by an external field $\mathbf{E}_{b}$. For longitudinal displacements this gives

$$
\mu \omega_{L}^{2} \mathbf{u}_{\mathbf{q}}=e^{*} \mathbf{E}_{b}
$$

where $\mu$ is the reduced mass and $\omega_{L}$ is the longitudinal phonon frequency. According to Eq.(7) this is equivalent to

$$
\mathbf{P}_{\mathbf{i}}=\frac{\left(e^{*}\right)^{2}}{\mu \omega_{L}^{2} \Omega} \mathbf{E}_{b}
$$


On the other hand, $\mathbf{E}+4 \pi \mathbf{P}=\mathbf{E}_{b}$ and $\mathbf{E}=\mathbf{E}_{b} / \varepsilon$. Thus

$$
\mathbf{P}=\frac{1}{4 \pi}\left(1-\frac{1}{\varepsilon}\right) \mathbf{E}_{b}
$$

where $\mathbf{P}=\mathbf{P}_{e}+\mathbf{P}_{\mathbf{i}}$, and $\mathbf{P}_{e}$ is the electronic polarizability. Eq. (12) is in general valid for fields with a harmonic time dependence $e^{i \omega t}$. In static conditions $(\omega=0$, $\varepsilon=\varepsilon(0))$, both $\mathbf{P}_{e}$ and $\mathbf{P}_{\mathbf{i}}$ contribute to $\mathbf{P}$, while, when $\omega \rightarrow \infty(\varepsilon=\varepsilon(\infty))$, the contribution of $\mathbf{P}_{\mathbf{i}}$ is negligible. Therefore, we can calculate $\mathbf{P}_{\mathbf{i}}$ as

$$
\mathbf{P}_{\mathbf{i}}=-\frac{1}{4 \pi}\left(\frac{1}{\varepsilon(0)}-\frac{1}{\varepsilon(\infty)}\right) \mathbf{E}_{b}
$$

The comparison between Eqs.(11) and (13) gives

$$
\left(e^{*}\right)^{2}=\mu \omega_{L}^{2}\left(\frac{1}{\varepsilon(\infty)}-\frac{1}{\varepsilon(0)}\right) \frac{\Omega}{4 \pi} .
$$

Eqs. (9) and (14) provide an expression of $\phi_{\mathbf{q}}$ in terms of $u_{\mathbf{q}}$, which, however, does not take into account the screening by the carriers. The latter can be easily included via linearized theories such as the Debye or Thomas-Fermi approximations.

In both cases, we have to replace $\frac{1}{q}$ in equation (9) by $\frac{q}{q^{2}+q_{s}^{2}}$ where $q_{s}^{2}$ is given either by Debye formula

$$
q_{D}^{2}=\frac{4 \pi e^{2}}{\varepsilon(\infty) k_{B} T} n_{0},
$$

valid at low carrier concentration $n_{0}$, or by the ThomasFermi expression

$$
q_{T F}^{2}=q_{D}^{2} \frac{3}{2} \frac{k_{B} T}{\epsilon_{F}}
$$

valid at high carrier concentrations, i.e when the Fermi level $\epsilon_{F}$ is located inside the band and its distance from the band edge is $>k_{B} T$, where $k_{B}$ is the Boltzmann constant and $\mathrm{T}$ is the absolute temperature. The electronphonon coupling is thus given by $-e \phi_{\mathbf{q}} \frac{e^{i \mathbf{q} \cdot \mathbf{R}}}{\sqrt{V}}$ which, with equation (9) modified for screening gives

$$
H_{e p}=i \frac{e q}{q^{2}+q_{s}^{2}} \frac{4 \pi e^{*}}{\Omega} u_{\mathbf{q}} \frac{e^{i \mathbf{q} \cdot \mathbf{R}}}{\sqrt{V}} .
$$

In fact, there are two sources of electron screening, one by the valence electrons, the other one by the excess carriers. For the first one the plasmon frequency is around 15 $\mathrm{eV}$, much higher than the typical optical phonon energies of order $50 \mathrm{meV}$. One can thus safely use static dielectric screening in this case which amounts to divide the phonon induced potential by the dielectric constant. The situation is not the same for the excess carriers since the squared plasma frequency scales as $n / \varepsilon m^{*}$ where $n$ is the excess electron concentration, $\varepsilon$ the dielectric constant and $m *$ the effective mass. For the weak concentrations considered here this can bring the corresponding plasma frequency in the same range as the phonon frequency. Qualitatively this would reduce $q_{s}$ (which represents the strength of the screening) with respect to its static value. In practice, we consider cases for which the static $q_{s}$ is negligible so that this effect can be discarded.

By combining Eqs. (6) and (14), we obtain

$$
\begin{aligned}
{\left[\Sigma_{P O P, \text { bulk }}^{\lessgtr}\left(\epsilon, \mathbf{r}_{1}, \mathbf{r}_{2}\right)\right] } & =\frac{M^{2}}{V}\left\{\left(N_{\text {pop }}+1\right) G^{\lessgtr}\left(\epsilon \pm \hbar \omega_{L}, \mathbf{r}_{1}, \mathbf{r}_{2}\right)\right. \\
& \left.+N_{\text {pop }} G^{\lessgtr}\left(\epsilon \mp \hbar \omega_{L}, \mathbf{r}_{1}, \mathbf{r}_{2}\right)\right\} \\
& \times \sum_{\mathbf{q}} \frac{e^{i \mathbf{q}\left(\mathbf{r}_{1}-\mathbf{r}_{2}\right)} q^{2}}{\left(q^{2}+q_{s}^{2}\right)^{2}},
\end{aligned}
$$

where $\Sigma \lessgtr$ is expressed in real space with coordinates $\mathbf{r}_{1}$ and $\mathbf{r}_{2}$ and $M^{2}=2 \pi \hbar \omega_{L} e^{2}\left(\frac{1}{\varepsilon(\infty)}-\frac{1}{\varepsilon(0)}\right)$.

\section{Extension to the case of a quantum well}

We consider first the case of a system in which phonons are confined only in the $z$ direction, between $z=0$ and $z=L_{z}$. In this work we have followed the general approach of Mori and Ando on electron-optical phonon interaction in heterostructures [51]. For a double heterostructure they show that one can separate the eigenmodes into confided LO modes with vanishing polarization field at the interface and interface modes decaying exponentially away from the interface. We concentrate here on the first category. A second point is related to the confinement effect on the phonon modes. This was shown to be important for acoustic modes due to their important dispersion curve [52]. However optical phonons exhibit almost no dispersion so that it is equivalent to treat them as Einstein oscillators, the confinement effect being negligible. As discussed previously we consider longitudinal PO displacements confined to the region extending between $z=0$ and $z=L_{z}$ which are solutions of $\varepsilon(\omega)=0$. We express the potential $\phi$ in terms of a complete set of bulk basis functions satisfying the correct boundary conditions at $z=0$ and $L_{z}$, i.e no polarization at the two interfaces:

$$
\phi(\mathbf{R})=\sum_{\mathbf{q}} \phi_{\mathbf{q}} \sqrt{\frac{2}{L_{z} A}} \sin \left(q_{z} z\right) \exp \left(i \mathbf{q}_{\|} \cdot \mathbf{r}_{\|}\right)
$$

where $\mathbf{R}=\left(\mathbf{r}_{\|}, z\right), q=\left(\mathbf{q}_{\|}, q_{z}\right)$, and $A$ is the transverse cross section and $q_{z}=\frac{n_{z} \pi}{L_{z}}$. As before, longitudinal modes correspond to $\mathbf{E}=-4 \pi \mathbf{P}_{\mathbf{i}}=-\nabla \phi$. As $\mathbf{P}_{\mathbf{i}}$ is still given by (7) each phonon mode $\mathbf{u}_{\mathbf{q}}(\mathbf{R})$ is related to $\phi_{\mathbf{q}}$ by

$$
\frac{4 \pi e^{*}}{\Omega} \mathbf{u}_{\mathbf{q}}(\mathbf{R})=\phi_{\mathbf{q}} \sqrt{\frac{2}{L_{z} A}}\left(\mathbf{q}_{n} \cos \left(q_{z} z\right)+i \mathbf{q}_{\|} \sin \left(q_{z} z\right)\right) e^{i \mathbf{q}_{\|} \cdot \mathbf{r}_{\|}} .
$$


From Eq.(20) it is straightforward to see that the eigenmodes $\mathbf{u}_{\mathbf{q}}(\mathbf{R})$ are orthogonal (i.e. $\mathbf{u}_{\mathbf{q}}(\mathbf{R}) \cdot \mathbf{u}_{\mathbf{q}}^{\prime}(\mathbf{R})=\delta_{q q^{\prime}}$ ) and

$$
u_{\mathbf{q}}^{2}=\frac{1}{V} \int\left|\mathbf{u}_{\mathbf{q}}(\mathbf{R})\right|^{2} d^{3} \mathbf{R}=\left(q \frac{\Omega}{4 \pi e^{*}} \phi_{\mathbf{q}}\right)^{2}
$$

with $q=\sqrt{q_{z}^{2}+q_{\|}^{2}}$. This means that

$$
\frac{\partial \phi(\mathbf{R})}{\partial u_{\mathbf{q}}}=\frac{4 \pi e^{*}}{\Omega q} \sqrt{\frac{2}{V}} \sin \left(q_{z} z\right) \exp \left(i \mathbf{q}_{\|} \cdot \mathbf{r}_{\|}\right)
$$

which leads exactly to the same expression for $\frac{\partial H_{e p}}{\partial u_{\mathbf{q}}}$ as Eq.(17) for the bulk, except for the replacement of $\sqrt{\frac{1}{V}} e^{i q_{z} z}$ by $\sqrt{\frac{2}{V}} \sin \left(q_{z} z\right)$.

This result can be extended to the case of a system where phonons are confined in two directions $x$ and $z$, by further replacing $e^{i q_{x} x}$ with $\sqrt{2} \sin \left(q_{x} x\right)$. In the following, we will mainly focus on this case, as it models the phonon confinement between the source and drain contacts and the top and bottom gate oxide in the DG MOSFET geometry presented in Section . Below, we report the corresponding expression of the lesser/greater than Green's function

$$
\begin{gathered}
{\left[\Sigma_{P O P}^{\lessgtr}\left(\epsilon, \mathbf{r}_{1}, \mathbf{r}_{2}\right)\right]=M^{2} \bar{G}^{\lessgtr}\left(\epsilon, \mathbf{r}_{1}, \mathbf{r}_{2}\right) I\left(\mathbf{r}_{1}, \mathbf{r}_{2}\right)} \\
\text { with : } \\
\bar{G}^{\lessgtr}\left(\epsilon, \mathbf{r}_{1}, \mathbf{r}_{2}\right)=\left(N_{\text {pop }}+1\right) G^{\lessgtr}\left(\epsilon \pm \hbar \omega_{L}, \mathbf{r}_{1}, \mathbf{r}_{2}\right) \\
+\quad N_{\text {pop }} G^{\lessgtr}\left(\epsilon \mp \hbar \omega_{L}, \mathbf{r}_{1}, \mathbf{r}_{2}\right), \\
\text { and : } \\
I\left(\mathbf{r}_{1} \mathbf{r}_{2}\right)=\frac{4}{V} \sum_{\mathbf{q}} \sin \left(q_{x} x_{1}\right) \sin \left(q_{x} x_{2}\right) \sin \left(q_{z} z_{1}\right) \sin \left(q_{z} z_{2}\right) \\
\times \frac{q^{2}}{\left(q^{2}+q_{s}^{2}\right)^{2}} e^{i q_{y}\left(y_{1}-y_{2}\right)}, \\
q_{x}=n_{x} \frac{\pi}{L_{x}}, \quad q_{z}=n_{z} \frac{\pi}{L_{z}}, \quad q_{y}=2 n_{y} \frac{\pi}{L_{y}} .
\end{gathered}
$$

\section{Scaling factor for 2D double-gate geometry}

In this Section, we will introduce the diagonal approximation for the PO phonon self-energies mapped on the discretized simulation domain of DG MOSFETs (Subsection 1 ). Then, we will derive an analytical expression for the scaling factor and we will provide simplified formulas allowing for a rapid numerical computation (Subsection and, respectively). We also report an analytical expression of the scaling factor for the case of a one-dimensional confinement and benchmark it value against previous numerical studies (Subsection ). For the sake of simplicity, the derivation is carried out for electrons, but the final results fully apply also to holes.

\section{The discretized diagonal approximation}

Let us denote by $\rho=(x, z)$ the position vector in the $x-z$ plane. We define a discretized version of $\Sigma_{P O P}^{\lessgtr}\left(y_{1}, \rho_{1} ; y_{2}, \rho_{2}\right)$ on a square lattice of parameter a as

$$
\begin{aligned}
\Sigma_{P O P, i, j}\left(y_{1}, y_{2}\right) & =\int \chi_{i}\left(\boldsymbol{\rho}_{1}\right) \Sigma_{P O P}^{\lessgtr}\left(y_{1}, \boldsymbol{\rho}_{1} ; y_{2}, \boldsymbol{\rho}_{2}\right) \chi_{j}\left(\boldsymbol{\rho}_{2}\right) \\
& =a^{2} \Sigma_{P O P}^{\lessgtr}\left(y_{1}, \mathbf{a}_{i} ; y_{2}, \mathbf{a}_{j}\right) .
\end{aligned}
$$

where $\chi_{i}(\boldsymbol{\rho})=1 / a$ is a normalized averaging function in the unit cell around the lattice site $\mathbf{a}_{i}$.

Since our system is uniform along the $y$ direction we first Fourier transform Eq.(23) and apply Eq.(24) with $\mathbf{a}_{i}=\mathbf{a}_{j}=\left(x_{i}, z_{i}\right)$. The diagonal elements of $\Sigma_{P O P}^{\lessgtr}$ then read as:

$$
\begin{array}{r}
{\left[\Sigma_{p o p, i i}^{\lessgtr}\left(\epsilon, k_{y}\right)\right]=\frac{4}{V} M^{2} a^{2} \sum_{\mathbf{q}} \bar{G}_{i, i}^{\lessgtr}\left(\epsilon, k_{y}-q_{y}\right)} \\
\sin ^{2}\left(q_{x} x_{i}\right) \sin ^{2}\left(q_{z} z_{i}\right) \frac{q^{2}}{\left(q^{2}+q_{s}^{2}\right)^{2}}
\end{array}
$$

We average the squared sine functions in Eq. (25) to $1 / 2$ and transform the discrete summation over $q_{x}$ and $q_{z}$ into an integral. Care must however be taken that, due to the confinement, $q_{x}$ and $q_{z}$ are discrete quantities (see Eq. (23)). We have found that an approximation procedure is

$$
\begin{aligned}
\frac{1}{L_{x} L_{z}} \sum_{\substack{q_{x}=\frac{n \pi}{L_{x}}>0 \\
q_{z}=\frac{m \pi}{L_{y}}>0}} \frac{q^{2}}{\left(q^{2}+q_{s}^{2}\right)^{2}} \simeq \frac{1}{\pi^{2}} \int_{0}^{q_{M}} d q_{x} \int_{0}^{q_{M}} d q_{z} \frac{q^{2}+q_{0}^{2}}{\left(q^{2}+q_{0}^{2}+q_{s}^{2}\right)^{2}} \\
q_{0}^{2}=\left(\frac{\pi}{2 L_{x}}\right)^{2}+\left(\frac{\pi}{2 L_{z}}\right)^{2}
\end{aligned}
$$

$q_{M}$ is the size of the Brillouin zone. After integration in polar coordinates, we finally obtain

$$
\begin{aligned}
{\left[\Sigma_{p o p, i i}^{\lessgtr}\left(\epsilon, k_{y}\right)\right]=} & \frac{M^{2} a^{2}}{4 \pi L_{y}} \sum_{q_{y}} \bar{G}_{i, i}^{\lessgtr}\left(\epsilon, k_{y}-q_{y}\right) \\
\times & \left\{\ln \frac{q_{M}^{2}+q_{y}^{2}+q_{0}^{2}+q_{s}^{2}}{\left(q_{y}^{2}+q_{0}^{2}+q_{s}^{2}\right)}-q_{s}^{2}\left(\frac{1}{q_{y}^{2}+q_{0}^{2}+q_{s}^{2}}\right.\right. \\
& \left.\left.-\frac{1}{q_{M}^{2}+q_{y}^{2}+q_{0}^{2}+q_{s}^{2}}\right)\right\}
\end{aligned}
$$

Based on this formula, in the next Subsection we will proceed to the calculation of the scaling factor. 
The scaling factor

We define the scaling factor $S$ by requiring that the diagonal approximation conserves the total scattering rates. Its Fourier transform is thus exact in the small $k$ limit. It has thus all the physical ingredients of the full second order expansion of the electron Greens function which considers that the phonons remain in thermal equilibrium. Here the numerical accuracy is checked by comparison with a previous 1D full calculation. A more detailed study of the validity of the local approximation is out of the scope of the present paper and will be the subject of a forthcoming work. We quantify the total scattering rate on a lattice site $a_{i}$ and at energy $\epsilon$ as $\sum_{j} \bar{\Gamma}_{i j}(\epsilon)$, where $\bar{\Gamma}=\lim _{k_{y} \rightarrow 0}\left[\Sigma_{p o p}^{<}\left(\epsilon, k_{y}\right)-\Sigma_{p o p}^{>}\left(\epsilon, k_{y}\right)\right]$ is the broadening function integrated over the $y$ direction. This expression takes into account all the phononassisted intersite hoppings. We therefore set

$$
S_{i}(\epsilon)=\frac{\sum_{j} \bar{\Gamma}_{i, j}(\epsilon)}{\bar{\Gamma}_{i, i}(\epsilon)}
$$

and we notice that $S$ is, in principle, site- and energydependent.

To proceed further we need to consider this broadening function in more detail.

According to Eq. (23),

$$
\Sigma_{P O P}^{<}(\epsilon)-\Sigma_{P O P}^{>}(\epsilon)=M^{2}\left[\bar{G}^{<}(\epsilon)-\bar{G}^{>}(\epsilon)\right] I
$$

In the spirit of the Boltzmann transport equation we will evaluate $S$ in equilibrium conditions. Therefore, we assume

$$
\begin{aligned}
& G^{<}(\epsilon)=i A(\epsilon) f(\epsilon) \\
& G^{>}(\epsilon)=-i A(\epsilon)[1-f(\epsilon)],
\end{aligned}
$$

where $f$ is the Fermi function and $A(\epsilon)$ is the spectral function. By injecting Eq. (30) into Eq. (29) and by using Eq. (23), we obtain

$$
\begin{aligned}
\bar{G}^{<}(\epsilon)-\bar{G}^{>}(\epsilon) & =i\left[N_{\text {pop }}+f\left(\epsilon+\hbar \omega_{L}\right)\right] A\left(\epsilon+\hbar \omega_{L}\right) \\
& +\left[N_{\text {pop }}+1-f\left(\epsilon-\hbar \omega_{L}\right)\right] A\left(\epsilon-\hbar \omega_{L}\right) \\
& =i \bar{A}(\epsilon)
\end{aligned}
$$

The continuous expression of $\bar{\Gamma}$ thus reads

$$
\begin{aligned}
\bar{\Gamma}\left(\epsilon, \boldsymbol{\rho}_{1}, \boldsymbol{\rho}_{2}\right) & =M^{2} \bar{A}\left(\epsilon, y_{1}, \boldsymbol{\rho}_{1}, y_{2}, \boldsymbol{\rho}_{2}\right) I\left(y_{1}, \boldsymbol{\rho}_{1}, y_{2}, \boldsymbol{\rho}_{2}\right) \\
& =M^{2} \sum_{q_{y}} \bar{A}\left(\epsilon, q_{y}, \boldsymbol{\rho}_{1}, \boldsymbol{\rho}_{2}\right) I\left(q_{y}, \boldsymbol{\rho}_{1}, \boldsymbol{\rho}_{2}\right)
\end{aligned}
$$

where the second equality follows by a Fourier transformation with respect to $\left(y_{1}-y_{2}\right)$ and by taking the limit $k_{y} \rightarrow 0$, and

$$
\begin{aligned}
\bar{A}\left(\epsilon, q_{y}, \boldsymbol{\rho}_{1}, \boldsymbol{\rho}_{2}\right) & =2 \pi \frac{2}{L_{x} L_{z}} \sum_{\mathbf{k}} \delta\left(\epsilon-\epsilon_{q_{y}}-\epsilon_{\mathbf{k}}\right) \\
& \times \sin \left(k_{z} z_{1}\right) \sin \left(k_{z} z_{2}\right) e^{i k_{x}\left(x_{1}-x_{2}\right)} .
\end{aligned}
$$

In order to simplify the notation, in the following we will refer to $\Gamma$,

$$
\Gamma\left(\epsilon, \boldsymbol{\rho}_{1}, \boldsymbol{\rho}_{2}\right)=M^{2} \sum_{q_{y}} \bar{A}\left(\epsilon, q_{y}, \boldsymbol{\rho}_{1}, \boldsymbol{\rho}_{2}\right) I\left(q_{y}, \boldsymbol{\rho}_{1}, \boldsymbol{\rho}_{2}\right),
$$

instead than to $\bar{\Gamma}$. In terms of $\Gamma_{i j}, S$ reads

$$
S_{i}=\sum_{j} \frac{\left[N_{p o p}+f\left(\epsilon+\hbar \omega_{L}\right)\right] \Gamma_{i j}\left(\epsilon+\hbar \omega_{L}\right)+\left[N_{p o p}+1-f\left(\epsilon-\hbar \omega_{L}\right)\right] \Gamma_{i j}\left(\epsilon-\hbar \omega_{L}\right)}{\left[N_{p o p}+f\left(\epsilon+\hbar \omega_{L}\right)\right] \Gamma_{i i}\left(\epsilon+\hbar \omega_{L}\right)+\left[N_{p o p}+1-f\left(\epsilon-\hbar \omega_{L}\right)\right] \Gamma_{i i}\left(\epsilon-\hbar \omega_{L}\right)} .
$$

Derivation of $\Gamma_{i i}$.

From Eqs. (33) and (34) with $\boldsymbol{\rho}_{1}=\boldsymbol{\rho}_{2}$, after averaging

$$
\begin{aligned}
\Gamma_{i i}(\epsilon) & =\frac{M^{2} a^{2}}{4 \pi L_{y}} \frac{2 \pi}{L_{x} L_{z}} \sum_{q_{y}, k_{x}, k_{z}} \delta\left(\epsilon-\epsilon_{q_{y}}-\epsilon_{k_{x}}-\epsilon_{k_{z}}\right) \\
& \times\left\{\ln \left(\frac{q_{M}^{2}+q_{y}^{2}+q_{0}^{2}+q_{s}^{2}}{\left(q_{y}^{2}+q_{0}^{2}+q_{s}^{2}\right)}\right)\right. \\
& \left.-q_{s}^{2}\left(\frac{1}{q_{y}^{2}+q_{0}^{2}+q_{s}^{2}}-\frac{1}{q_{M}^{2}+q_{y}^{2}+q_{0}^{2}+q_{s}^{2}}\right)\right\} .
\end{aligned}
$$


We notice that performing the average over $z$ actually removes the dependence of the diagonal elements of $\Gamma$ on the site index $i$. In Eq. (36) the energies $\epsilon_{k_{z}}$ are quantized with $k_{z}=\frac{n \pi}{L_{z}}>0$ while $\epsilon_{q_{y}}$ and $\epsilon_{k_{x}}$ are continuous quantities. We can thus replace the sum $\frac{2 \pi}{L_{x} L_{z}} \sum_{q_{y}, k_{x}}$ by an integral $\frac{1}{2 \pi} \int d k_{x} d q_{y}$. We use polar coordinates to transform it to $\frac{1}{2 \pi} \int \rho d \rho d \theta$ with $\rho^{2}=k_{x}^{2}+q_{y}^{2}$ and $q_{y}^{2}=\rho^{2} \cos ^{2} \theta$. With the further change of variable $\epsilon_{\rho}=\frac{\hbar^{2}}{2 m^{*}} \rho^{2}, \rho d \rho=\frac{m^{*}}{\hbar^{2}} d \epsilon_{\rho}$, we see that the delta function gives 1 if $\epsilon-\epsilon_{k_{z}}>0$. Finally, by averaging the functions $\cos ^{2} \theta$ to $1 / 2$, we obtain:

$$
\begin{gathered}
\Gamma_{i i}(\epsilon)=\frac{M^{2} a^{2} m^{*}}{4 \pi L_{z} \hbar^{2}} \sum_{k_{z}<k_{\epsilon}}\left\{\ln \left(\frac{q_{M}^{2}+q_{0 z}^{2}(\epsilon)}{q_{0 z}^{2}(\epsilon)}\right)\right. \\
\left.-q_{s}^{2}\left(\frac{1}{q_{0 z}^{2}(\epsilon)}-\frac{1}{q_{M}^{2}+q_{0 z}^{2}(\epsilon)}\right)\right\}
\end{gathered}
$$

with $q_{0 z}^{2}(\epsilon)=q_{0}^{2}+q_{s}^{2}+\frac{k_{\epsilon}^{2}-k_{z}^{2}}{2}, q_{0}^{2}$ defined by Eq.(26), and $k_{\epsilon}^{2}-k_{z}^{2}>0$.

The strong confinement along $z$ in a DG MOSFET geometry implies widely separated subbands. Therefore, only the lowest subband, corresponding to $k_{z}=\pi / L_{z}$, contributes to the sum in Eq. (37), and we can set $\left(k_{\epsilon}^{2}-\right.$ $\left.k_{z}^{2}\right) / 2=m^{*} / \hbar^{2}\left(\epsilon-\epsilon_{1}\right)$, where $\epsilon_{1}$ is the energy of the bottom of the lowest subband.

It is interesting to cast this result under a more usual form, in terms of parameter $\alpha$ which mesures the strength of the Fröhlich interaction

$$
\alpha=\frac{e^{2}}{\hbar}\left(\frac{m^{*}}{2 \hbar \omega_{L}}\right)^{1 / 2}\left(\frac{1}{\varepsilon_{\infty}}-\frac{1}{\varepsilon_{0}}\right)
$$

From Eq.(18) we can write:

$$
\frac{M^{2}}{\pi} \frac{m^{*}}{\hbar^{2} k_{L}}=2 \alpha \hbar \omega_{L} \quad \text { with } \quad k_{L}=\left(\frac{2 m^{*}}{\hbar^{2}} \hbar \omega_{L}\right)^{1 / 2} .
$$

By using Eq.(39) and by setting $k_{z}=k_{1}=\pi / L_{z}$, we obtain

$$
\begin{array}{r}
\Gamma_{i i}(\epsilon)=\frac{\alpha \hbar \omega_{L}}{2 \pi}\left(k_{1} k_{L} a^{2}\right)\left\{\ln \left(\frac{q_{M}^{2}+q_{0 z}^{2}}{q_{0 z}^{2}}\right)\right. \\
\left.-q_{s}^{2}\left(\frac{1}{q_{0 z}^{2}}-\frac{1}{q_{M}^{2}+q_{0 z}^{2}}\right)\right\}
\end{array}
$$

with $q_{0 z}^{2}(\epsilon)=q_{0}^{2}+q_{s}^{2}+\frac{k_{\epsilon}^{2}-k_{1}^{2}}{2}$.

$$
\text { Derivation of } \sum_{j} \Gamma_{i j}(\epsilon) \text {. }
$$

Here it is useful to map the sum over $j$ into an integral:

$$
\sum_{j} \Gamma_{i j}=a^{2} \sum_{j} \Gamma\left(\mathbf{a}_{i}, \mathbf{a}_{j}\right)=\int \Gamma\left(\mathbf{a}_{i}, \mathbf{r}\right) d \mathbf{r}
$$

From Eqs. (32), (33) and (41) we obtain

$$
\begin{aligned}
\sum_{j} \Gamma_{i j} & =M^{2} \frac{2}{L_{z}} \int_{-\infty}^{+\infty} d k_{x} \sum_{k_{z}} \int d x d z \sum_{k_{z}, q_{y}} \delta\left(\epsilon-\epsilon_{q_{y}}-\epsilon_{\mathbf{k}}\right) \\
& \times \frac{4}{V} \sin \left(k_{z} z_{i}\right) \sin \left(k_{z} z\right) e^{i k_{x}\left(x_{i}-x\right)} \\
& \times \sum_{q_{x} q_{z}} \sin \left(q_{z} z_{i}\right) \sin \left(q_{z} z\right) \sin \left(q_{x} x_{i}\right) \sin \left(q_{x} x\right) \frac{q^{2}}{\left(q^{2}+q_{s}^{2}\right)^{2}}
\end{aligned}
$$

In this expression, the integrals over $z$ and $x$ lead to $\delta_{k_{z}, q_{z}}$ and $\delta_{k_{x},-q_{x}}-\delta_{k_{x}, q_{x}}$, respectively. We obtain:

$$
\sum_{j} \Gamma_{i j}=M^{2} \frac{4 \pi}{V} \sum_{\mathbf{q}} \delta\left(\epsilon-\epsilon_{\mathbf{q}}\right) \sin ^{2}\left(q_{z} z_{i}\right) \sin ^{2}\left(q_{x} x_{i}\right) \frac{q^{2}}{\left(q^{2}+q_{s}^{2}\right)^{2}},
$$

where $q_{x}=\frac{n_{x} \pi}{L_{x}}>0, q_{z}=\frac{n_{z} \pi}{L_{z}}>0, q_{y}=\frac{2 \pi n_{y}}{L_{y}} \lessgtr 0$.

After averaging the squared sine functions in Eq. (43) to $1 / 2$ and transforming the sum over $q_{x}$ and $q_{y}$ into an integral, by following steps analogous to the derivation of Eq. (37), we arrive at

$$
\sum_{j} \Gamma_{i j}=\frac{M^{2}}{\pi} \frac{m^{*}}{\hbar^{2}} \frac{\pi}{L_{z}} \sum_{k_{z}} \frac{k_{\epsilon}^{2}}{k_{\epsilon}^{2}+q_{s}^{2}} .
$$

As for $\Gamma_{i i}$, by averaging over $x$ and $z$, we have obtained an expression independent of the lattice site index $i$. In terms of $\alpha, k_{1}$ and $k_{L}$ defined before, and by restricting the sum over $k_{z}$ to the first subband, we finally obtain:

$$
\sum_{j} \Gamma_{i j}=2 \alpha \hbar \omega_{L} \frac{k_{1} k_{L} k_{\epsilon}^{2}}{\left(k_{\epsilon}^{2}+q_{s}^{2}\right)^{2}}
$$

Numerical value of the scaling factor in the case of a quantum well

The numerical evaluation of the scaling factor in Eq. (35) can be simplified by taking into account that the transport occurs only through the lowest subband. We first consider two different limits:

1. High charge-densities: the transport occurs at energies close to the Fermi level $\epsilon_{F}$, which lies within the lowest confined subband: $\epsilon_{F}>\epsilon_{1}$. As $\epsilon_{1} \gg$ $\hbar \omega_{L} \simeq 30 \mathrm{meV}$, we can write

$$
S=\sum_{j} \frac{\Gamma_{i j}\left(\epsilon_{F}\right)}{\Gamma_{i i}\left(\epsilon_{F}\right)}
$$

By using Eqs. (40) and (45), and taking into account that $q_{M} \gg q_{0 z}$, we find

$$
S=\frac{2 \pi}{\left(k_{F} a\right)^{2}} \frac{1}{\left(1+\frac{q_{s}^{2}}{k_{F}^{2}}\right)^{2}\left[\ln \left(\frac{q_{M}}{q_{0 z}}\right)-\frac{1}{2} \frac{q_{s}^{2}}{q_{0 z}^{2}}\right]},
$$

where $k_{F}=\sqrt{\left(2 m^{*} \epsilon_{F}\right)} / \hbar$ is the Fermi wave vector. 
TABLE II. Numerical values of $S$ as a function of $L_{z}$.

\begin{tabular}{lcccccccc}
\hline \hline$L_{z}$ & 2.0 & 2.2 & 2.4 & 2.6 & 2.8 & 3.0 & 3.2 & 3.4 \\
$S$ & 81.7 & 95.9 & 111.1 & 127.3 & 144.5 & 162.6 & 181.7 & 201.8 \\
\hline
\end{tabular}

\begin{tabular}{lllllllll}
$S$ & 81.7 & 95.9 & 111.1 & 127.3 & 144.5 & 162.6 & 181.7 & 201.8 \\
\hline
\end{tabular}

2. Low charge densities: $\epsilon_{F}$ is below the bottom of the lowest confined subband, and the transport energies are within few $k_{B} T$ from $\epsilon_{1}$. Therefore, also in this case we can use Eq. (47), provided $k_{F}$ is replaced with $k_{1}=\sqrt{\left(2 m^{*} \epsilon_{1}\right)} / \hbar=\pi / L_{z}$.

For both high and low charge densities, it can be shown that $q_{0} z \simeq k_{1}$ and $q_{s} \ll k_{1}$, which allows Eq. (47) to be further simplified. In the case of low charge density we find

$$
S=\frac{2 \pi}{\left(k_{1} a\right)^{2}} \frac{1}{\ln \left(\frac{2 q_{M}}{k_{1}}\right)} .
$$

In the considered systems, where the confinement energy contribution dominates over those associated to the unconfined directions, $k_{F} \simeq k_{1}$, and Eq. (48) is also valid in the high charge density case. Moreover, it is easy to argue that this result also applies to charge density values intermediate between the considered limits. Eq. (48) predicts that $S$ is essentially independent of the effective mass, and dominated by $k_{1}$, or, equivalently, by $L_{z}$. To exemplify, Table II reports the numerical values of $S$ corresponding to several different values of $L_{z}$.

Based on interaction self-energies (Eqs.(3-5) (27) and (48)), physical quantities like carrier density and electronic current can be calculated [23]. NEGF equations are then self-consistently with Poisson equation to determine the actual electrostatic potential in the active region.

\section{The scaling factor for the $1 D$ case}

It is obviously of strong interest to test our method against the results of numerical computations able to take into account the full electron-PO phonon scattering selfenergy.

In Ref. 39, full self-energy computations were performed in the case of GaAs-AlAs resonant tunneling diodes and compared to a $1 \mathrm{D}$ diagonal approximation along the direction of the current. In this study, it was unambiguously shown that the full self-energy results could be recovered within a diagonal approach by using a scaling factor $S=12$. By following an analogous approach to that previously described in the case of $2 \mathrm{D}$ confinement, we find that the equivalent of Eq.(48) for a system confined along a single direction is

$$
S=\frac{4}{\pi a \sqrt{k_{F}^{2}+k_{L}^{2}}}
$$
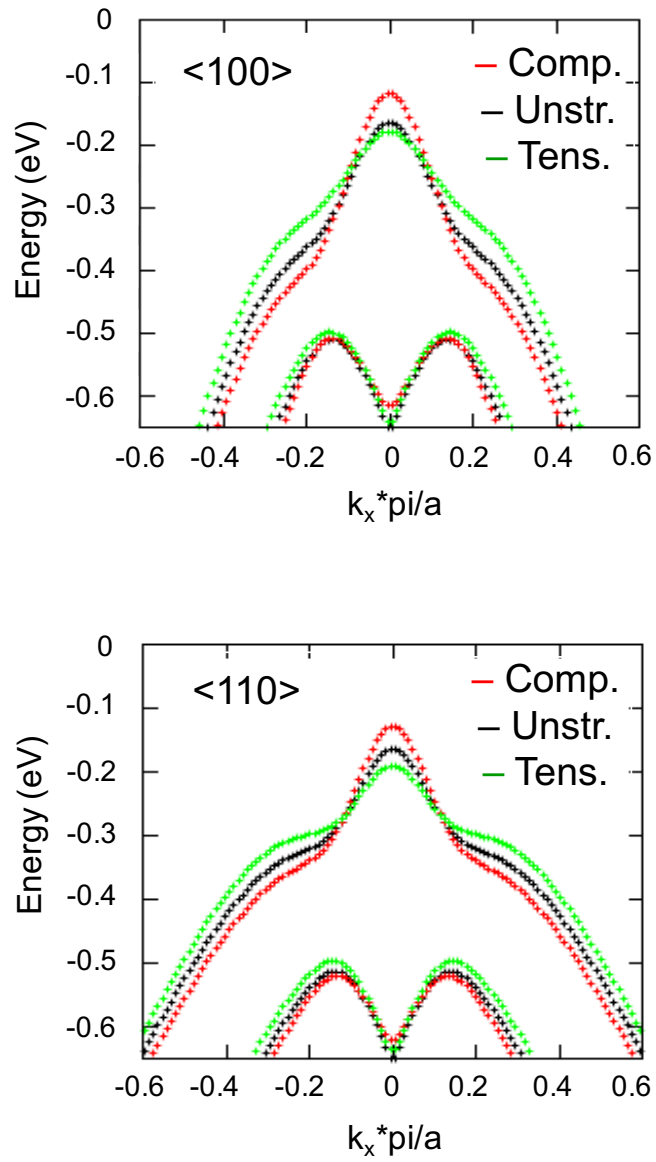

FIG. 2. Impact of uniaxial compressive and tensile strain, corresponding to a deformation $|\epsilon|=3 \%$, on the valence band structure of a $2.4 \mathrm{~nm}$-thick InAs film. Both the strain and the band structure are along the direction $<100>$ (top), and $<110>$ (bottom).

with $k_{L}$ defined by equation (39). In the case of GaAs at low temperature, for small electron concentration $\left(k_{F} \ll\right.$ $k_{L}$ ), with $\hbar \omega_{L}=33 \mathrm{meV}$ and a discretization parameter of $a=4 \AA$, this gives $S=12.7$, in excellent agreement with the results of Ref.39.

\section{SIMULATIONS OF DG P-TYPE TRANSISTORS}

Based on the model developed in Section, we now investigate the performance of III-V DG p-MOSFETs through self-consistent NEGF simulations, which couple the kinetic equations introduced in Section with the twodimensional Poisson equation in the device cross-section.

The thickness of the III-V film is assumed to be $T_{S C}=2.4 \mathrm{~nm}$ and the source and drain extensions (see Fig. 1) are p-doped with a concentration of $8 \times 10^{19}$ $\mathrm{cm}^{-3}$. We consider as channel materials InAs, InSb and GaSb, as they provide the highest hole bulk mobilities. Performances are investigated as a function 


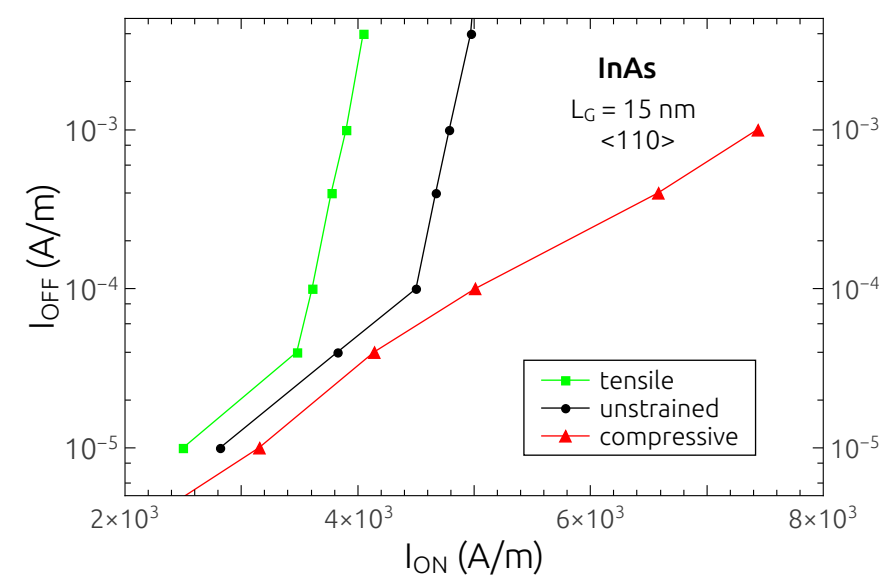

FIG. 3. Impact of strain $|\epsilon|=3 \%$ on the ballistic $\mathrm{I}_{\mathrm{OFF}} v s \mathrm{I}_{\mathrm{ON}}$ characteristics of $\mathrm{a}<110>$-oriented InAs DG MOSFET with $L_{G}=15 \mathrm{~nm}$.

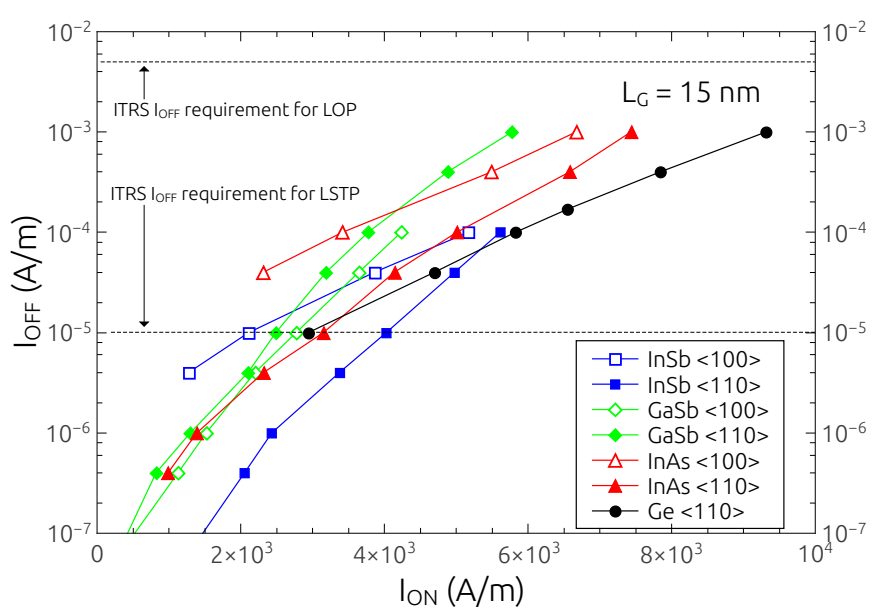

FIG. 4. Ballistic IOFF vs ION characteristics of InAs, InSb, GaSb and Ge DG MOSFETs with different orientations, under compressive strain $(|\epsilon|=3 \%)$. In all the cases $L_{G}=15 \mathrm{~nm}$.

of two different crystallographic orientations, $<100>$ and $\langle 110\rangle$, and strain constraints (uniaxial tensile and compressive strain), and benchmarked against analogous $\mathrm{Si}$ and Ge devices. All the simulations were carried out with a source to drain voltage $\mathrm{V}_{\mathrm{DS}}=-0.6 \mathrm{~V}$. Acoustic- and optical phonon-electron interactions were described through the self-energies defined in Eqs.(3-5), by using the deformation potential values in Ref. 53 . The electron-PO phonon scattering has been included through the diagonal self-energy (27), rescaled by a factor $S=111.1$ (see Table II).

Figure 2 shows how a uniaxial compressive and tensile strain along either the $<100>$ or $<110>$ direction, modifies the valence band structure of a $2.4 \mathrm{~nm}$ thick InAs film. The main impact on the transport is expected to derive from the modifications undergone by first subband, which is widely separated from the others

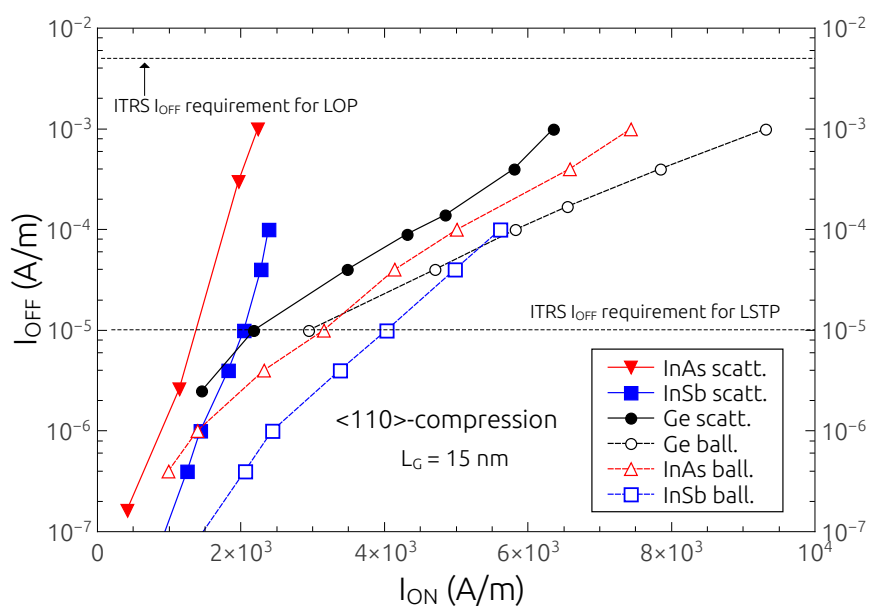

FIG. 5. $\mathrm{I}_{\mathrm{OFF}}$ vs $\mathrm{I}_{\mathrm{ON}}$ characteristics of $<110>$-oriented InAs, InSb and Ge DG MOSFETs under compressive strain $(|\epsilon|=3 \%)$, and in the presence of electron-phonon scattering. In all the cases $L_{G}=15 \mathrm{~nm}$.

TABLE III. Effective mass values $\left(\times m_{0}\right)$ extracted from the first valence subband.

\begin{tabular}{lccc}
\hline \hline & InAs & InSb & GaSb \\
\hline$<110>$ direction & & & \\
\hline Unstrained & 0.041 & 0.067 & 0.061 \\
Comp. & 0.032 & 0.0469 & 0.044 \\
Tens. & 0.0548 & 0.184 & 0.107 \\
\hline$<100>$ direction & & & \\
\hline Unstrained & 0.412 & 0.059 & 0.061 \\
Comp. & 0.030 & 0.042 & 0.047 \\
Tens. & 0.059 & 0.140 & 0.094 \\
\hline \hline
\end{tabular}

due to the strong confinement, and therefore dominates the transport. Both in the $\langle 100\rangle$ and $<110\rangle$ directions, a compressive strain induces a reduction of the effective mass value at $k_{x}=0$, while a tensile strain leads to an increase of it. We found the same behavior in the case of InSb and GaSb. The values of the effective mass extracted for the three materials as function of the crystallographic orientation and strain are reported in Table III.

In order to assess the impact of strain on the $\mathrm{I}_{\mathrm{ON}} / \mathrm{I}_{\mathrm{OFF}}$ ratio, we computed, for each strain constraint, transport direction and material, the OFF-state current $\mathrm{I}_{\mathrm{OFF}}$ as a function of the ON-state current $\mathrm{I}_{\mathrm{ON}}$. To isolate the effect of the strain from those due to the modulation of the electron-phonon interaction, a ballistic transport regime was assumed.

The case of the InAs MOSFET in the $<110>$ orientation with $L_{G}=15 \mathrm{~nm}$ is shown in Figure 3. The shift of the curves reflects the value of the first subband effective mass: devices with a lower effective mass tend to have better $\mathrm{I}_{\mathrm{ON}} / \mathrm{I}_{\mathrm{OFF}}$ ratios, and therefore $\mathrm{I}_{\mathrm{OFF}}-\mathrm{I}_{\mathrm{ON}}$ characteristics shifted toward higher $\mathrm{I}_{\mathrm{ON}}$ values. This trend remains unchanged for all the considered materials and 
transport directions (not shown).

According to these results, in the following we restrict the focus to compressively strained devices. Fig.4 illustrates the $\mathrm{I}_{\mathrm{OFF}}-\mathrm{I}_{\mathrm{ON}}$ characteristics of the InAs, InSb and GeSb MOSFETs. The results for a $<110>$-oriented, compressively strained MOSFET with the channel in Ge, which has been shown to represent a better option than $\mathrm{Si}$ for DG devices at $L_{G}=15 \mathrm{~nm}$ [41], are also included. Our results indicate that Ge-based devices can outperform the others with respect to low operating power (LOP) requirements. Among the considered III-V devices, the $<100>$-oriented InAs and InSb MOSFETs appear to provide the best performance for LOP and low standby power (LSTP) applications, respectively.

In Figure 5, we plot the $\mathrm{I}_{\mathrm{OFF}}-\mathrm{I}_{\mathrm{ON}}$ characteristics in the presence of electron-phonon scattering for the InAs, InSb and Ge MOSFETs. The corresponding ballistic curves are also plotted for comparison. As expected, for all the materials, the impact of the electron-phonon scattering is stronger for high $\mathrm{I}_{\mathrm{ON}}$ values. However, in the case of Ge, the effect is significantly weaker. More precisely, for a IOFF current of $10^{-4} \mathrm{~A} / \mathrm{m}$, the electron-phonon interaction induces a reduction of the ON-current of about $65 \%$ and $58 \%$ in InAs and InSb, respectively, while this value amounts to $24 \%$ in Ge. The relative importance of the electron-PO phonon scattering in determining this difference can be quantified by evaluating the $\mathrm{I}_{\mathrm{ON}}$ reduction in the III-V devices due only to acoustic and non-polar optical phonons. In these conditions, for the InAs devices we found a $\mathrm{I}_{\mathrm{ON}}$ reduction of around $25 \%$. Similar values are expected for the InSb device. Thus, our results indicate that electron-PO phonon interactions can strongly disadvantage III-V MOSFETs with respect to analogous non-polar material devices. In Fig. 5, this directly translates in a widening of the performance gap between the III-V and Ge devices with respect to ballistic transport conditions. It turns out that the Ge MOSFET can largely outperform the III-V counterparts for LOP applications. InAs and InSb devices are competitive only for LSTP applications, as the impact of the electron-phonon scattering reduces due to the smaller current.

A substantially different scenario arises when shorter gate lengths are considered. The results we obtained by repeating all the previous simulations for $L_{G}=7 \mathrm{~nm}$ indicate that the $<110>$-oriented GaSb MOSFET exhibits in this case the best $\mathrm{I}_{\mathrm{ON}} / \mathrm{I}_{\mathrm{OFF}}$ performance (not shown). Fig. 6 illustrates the $\mathrm{I}_{\mathrm{ON}}-\mathrm{I}_{\mathrm{OFF}}$ characteristics of this device with and without strain, in the ballistic regime and in the presence of electron-phonon scattering. These results are compared with those obtained for a $<100>$ oriented, tensile-strained Si MOSFET, which, at this gate length scale, exhibits better performance with respect to the corresponding Ge-based device [41]. In the ballistic regime, we see that the Si device clearly outperforms all the III-V devices, for both LOP and LSTP applications. Indeed, the larger effective mass $\left(\mathrm{m}^{*}=0.3 \times \mathrm{m}_{0}\right)$

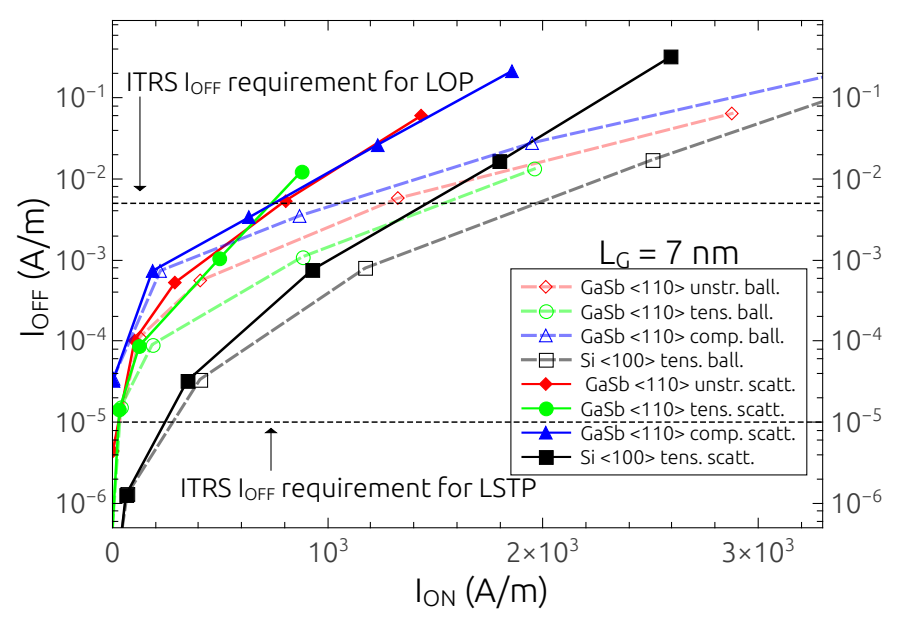

FIG. 6. $\mathrm{I}_{\mathrm{OFF}}$ vs $\mathrm{I}_{\mathrm{ON}}$ characteristics for $<110>$-oriented, compressively strained, tensile strained and unstrained GaSb MOSFETs, compared with the corresponding $<100>-$ oriented, tensile strained Si device. $L_{G}=7 \mathrm{~nm}$.

becomes a significant advantage at this channel length, since it mitigates the OFF-current degradation due to the source-to-drain tunneling. [40, 41] For the same reason, the $<110>$-oriented, tensile-strained GaSb MOSFET, in which the first subband effective mass $\left(\mathrm{m}^{*}=0.107 \times \mathrm{m}_{0}\right)$ is larger than for the other GaSb devices, exhibits the second best performance.

As in the longer channel case, the inclusion of electronphonon scattering leads to a larger current degradation in III-V devices. This results in a significant improvement of the Si MOSFET performance with respect to the GaSb device, which exhibits, at the same $\mathrm{I}_{\mathrm{ON}}$, an $\mathrm{I}_{\mathrm{ON}} / \mathrm{I}_{\mathrm{OFF}}$ ratio smaller of at least one order of magnitude.

\section{CONCLUSION}

In this work, we proposed a physically sound and numerically efficient NEGF quantum transport methodology to treat non-local polar optical phonon scattering. The methodology is based on a diagonal (i.e. local) selfenergy approximation, in which long range effects are incorporated through the introduction of a scaling factor. We derived a simple analytical expression of this factor for a 2D phonon quantum well. An analytical expression for a 1D well was also provided and checked against previous numerical studies. Based on a 8-band $\mathrm{k} \cdot \mathrm{p}$ Hamiltonian we used this approach to assess the performance of p-type III-V DG transistors for different channel materials, crystallographic orientations and applied strains. Our simulation results indicate that III-V transistors are hardly expected to outperform their Ge and Si counterparts and that the strong impact of polar optical phonon scattering in such materials is one the main reasons. Ac- 
cordingly, our results also highlights the need, even in the case of transistors with very scaled gate lengths, to accurately model the electron-PO phonon interaction in order to obtain reliable predictions of the device operation.

* michel.lannoo@im2np.fr

† bescond@iis.u-tokyo.ac.jp

[1] K. J. Kuhn, "Considerations for ultimate CMOS," IEEE Trans. Electron Devices 59, 1813 (2012).

[2] J.P. Colinge, "Multiple-gate SOI MOSFETs," Solid States Electron. 48, 897 (2004).

[3] J.-L. Autran, D. Munteanu, M Bescond, M Houssa, and A Said, "A simulation analysis of FIBL in decananometer double-gate MOSFETs with high- $\kappa$ gate dielectrics," J. Non-Crys. Solids 351, 1897 (2005).

[4] R. Kim, T. Rakshit, R. Rotlyar, S. Hasan, and C. E. Weber, "Effects of surface orientation on the performance of idealized III - V thin-body ballistic n - MOSFETs," IEEE Electron Device Lett. 32, 746 (2011).

[5] M. Xu, R. Wang, and P.D. Ye, "GaSb inversion-mode PMOSFETs with atomic-layer-deposited $\mathrm{Al}_{2} \mathrm{O}_{3}$ as gate dielectric," IEEE Electron Device Lett. 32, 883 (2011).

[6] H Fang, S. Chuang, K. Takei, H. S. Kim, E. Plis, C.-H. Liu, S. Krishna, Y.-L. Chueh, and A. Javey, "Ultrathinbody high-mobility InAsSb-on-insulator field-effect transistors," IEEE Electron Device Lett. 33, 504 (2012).

[7] J. A. Alamo, "Nanometre-scale electronics with III - V compound semiconductor," Nature 479, 317 (2012).

[8] K. Tomioka, M. Yoshimura, and T. Fukui, "A III - V nanowire channel on silicon for high-performance vertical transistors," Nature 488, 189 ((2012)).

[9] D. H. Kim and J. A. del Alamo, "30 nm InAs pseudomorphic HEMTs on an InP substrate with a current-gain cutoff frequency of $628 \mathrm{GHz}$," IEEE Electron Device Lett. 29, 830 (2008).

[10] M. V. Fischetti, L. Wang, B. Yu, C. Sachs, P. M. Asbeck, Y. Taur, and M. Rodwell, "Simulation of electron transport in high-mobility MOSFETs: Density of states bottleneck and source starvation," in International Electron Devices Meeting (IEDM) (IEEE, 2007) p. 109.

[11] "IRDS home page: https://irds.ieee.org/,".

[12] M. Heyns and W. Tsai, "Ultimate scaling of CMOS logic devices with Ge and III-V materials," Mrs bulletin 34, 485-492 (2009).

[13] S. Suthram, Y. Sun, P. Majhi, I. Ok, H. Kim, H. R. Harris, N. Goel, S. Parthasarathy, A. Koehler, T. Acosta, and others, "Strain additivity in III-V channels for CMOSFETs beyond 22nm technology node," in VLSI Technology, 2008 Symposium on (IEEE, 2008) pp. 182183.

[14] J. Kim and M. V. Fischetti, "Electronic band structure calculations for biaxially strained $\mathrm{Si}, \mathrm{Ge}$, and III-V semiconductors," J. Appl. Phys. 108, 013710 (2010).

[15] S. H. Park, Y. Liu, N. Kharche, M. S. Jelodar, G. Klimeck, M. S. Lundstrom, and M. Luisier, "Performance comparisons of III-V and strained-Si in planar FETs and nonplanar FinFETs at ultrashort gate length (12 nm)," IEEE Trans. Electron Devices 59, 2107 (2012).

[16] S. W. Bedell, A. Khakifirooz, and D. K. Sadana, "Strain scaling for CMOS," MRS Bulletin 39, 131 (2014).
[17] M. Yokoyama, T. Yasuda, H. Takagi, N. Miyata, Y. Urabe, H. Ishii, H. Yamada, N. Fukuhara, M. Hata, M. Sugiyama, Y. Nakano, M. Takenaka, and S. Takagi, "III-V-semiconductor-on-insulator nchannel metal-insulator-semiconductor field-effect transistors with buried $\mathrm{Al}_{2} \mathrm{O}_{3}$ layers and sulfur passivation: Reduction in carrier scattering at the bottom interface," Applied Physics Letters 96, 142106 (2010).

[18] A. Pethe, T. Krishnamohan, D. Kim, S. Oh, H.-S. P. Wong, and K. Saraswat, in 2006 16th Biennial University/Government/Industry Microelectronics Symposium (IEEE, 2006) pp. 47-50.

[19] G. Doornbos and M. Passlack, "Benchmarking of III-V n-MOSFET maturity and feasibility for future CMOS," IEEE Electron Device Letters 31, 1110-1112 (2010).

[20] R. Kim, U. E. Avci, and I. A. Young, "CMOS performance benchmarking of $\mathrm{Si}$, InAs, GaAs, and Ge nanowire $\mathrm{n}$-and pMOSFETs with $\mathrm{Lg}=13 \mathrm{~nm}$ based on atomistic quantum transport simulation including strain effects," in 2015 IEEE International Electron Devices Meeting (IEDM) (IEEE, 2015) pp. 34-1.

[21] M. Shin, "Full-quantum simulation of hole transport and band-to-band tunneling in nanowires using the k.p method," J. Appl. Phys. 106, 054505 (2009).

[22] N. Cavassilas, N. Pons, F. Michelini, and M. Bescond, "Multiband quantum transport simulations of ultimate p-type double-gate transistors: Influence of the channel orientation," Applied Physics Letters 96, 102102 (2010).

[23] N. Cavassilas, F Michelini, and M Bescond, "Multiband quantum transport simulations of ultimate p-type double-gate transistors: Effects of hole-phonon scattering," J. Appl. Phys. 109, 073706 (2011).

[24] V.-H. Nguyen, Y.-M. Niquet, F. Triozon, I. Duchemin, O. Nier, and D. Rideau, "Quantum modeling of the carrier mobility in FDSOI devices," IEEE Trans. Electron Devices 61, 3096 (2014).

[25] Y. Lee, M. Bescond, N. Cavassilas, D. Logoteta, L. Raymond, M. Lannoo, and M. Luisier, "Quantum treatment of phonon scattering for modeling of three-dimensional atomistic transport," Phys. Rev. B (R) 95, 201412 (2017).

[26] S. Datta, Electronic Transport in Mesoscopic Systems (Cambridge University Press, Cambridge, U.K., 1995).

[27] D. K. Ferry and S. M. Goodnick, Transport in Nanostructures (Cambridge University Press, Cambridge, U.K., 1997).

[28] H. Haug and A.-P. Jauho, Quantum Kinetics in Transport and Optics of Semiconductors, vol. 123 of Springer Series in Solid-State Sciences (Springer, Berlin, New York, 1996).

[29] G. D. Mahan, Many-Particle Physics (Plenum, New York, 1990).

[30] M. Luisier and G. Klimeck, "Atomistic full-band simulations of silicon nanowire transistors: Effects of electronphonon scattering," Phys. Rev. B. 80, 155430 (2009).

[31] M. P. Lopez Sancho, J. M. Lopez Sancho, J. M. L. Sancho, and J. Rubio, "Highly convergent schemes for the calculation of bulk and surface Green functions," J. Phys. F, Metal Phys. 15, 851 (1985).

[32] A. Svizhenko and M. P. Anantram, "Role of scattering in nanotransistors," IEEE Trans. Electron Dev. 50, 1459 (2003).

[33] S. Jin, Y. J. Park, and H. S. Min, "A three-dimensional simulation of quantum transport in silicon nanowire tran- 
sistor in the presence of electron-phonon interactions," J. Appl. Phys. 99, 123719 (2006).

[34] M. Bescond, H. Carrillo-Nuñez, S. Berrada, N. Cavassilas, and M. Lannoo, "Size and temperature dependence of the electronphonon scattering by donors in nanowire transistors," Solid-State Electron. 122, 1-7 (2016).

[35] N. Cavassilas, F. Michelini, and M. Bescond, "On the local approximation of the electronphoton interaction selfenergy," J. Comp. Electron. 15, 1233 (2016).

[36] H. Fröhlich, "Theory of electrical breakdown in fonic crystals," in Proc. R. Soc. London, Ser. A ((1937)) p. 230.

[37] J. Appel, "Polarons," Solid State Physics 21, 193 (1968).

[38] H. Ehrenreich, "Electron scattering in InSb," J. Phys. Chem. Solids 2, 131 (1957).

[39] G. Klimeck, R. Lake, C. Fernando, R. Bowen, D. Blanks, M. Leng, T. Moise, Y. Kao, and W. Frensley, "Numerical approximations for polar optical phonon scattering in resonant tunneling diodes," Quantum Devices and Circuits. Imperial Press, London (1996).

[40] E. Dib, M. Bescond, N. Cavassilas, F. Michelini, L. Raymond, and M. Lannoo, "Theoretical comparison of Si, $\mathrm{Ge}$, and GaAs ultrathin p-type double-gate metal oxide semiconductor transistors," J. Appl. Phys. 114, 083705 (2013).

[41] M. Moussavou, N. Cavassilas, E. Dib, and M. Bescond, "Influence of uniaxial strain in $\mathrm{Si}$ and Ge p-type doublegate metal-oxide-semiconductor field effect transistors," J. Appl. Phys. 118, 114503 (2015).

[42] A. Nainani, B. R. Bennett, J. B. Boos, M. G. Ancona, and K. C. Saraswat, "Enhancing hole mobility in III-V semiconductors," J. Appl. Phys. 111, 103706 (2012).

[43] T. B. Bahder, "Eight-band k.p model of strained zincblende crystals," Phys. Rev. B 41, 11992 (1990).
[44] G. L. Bir and G. E. Pikus, Symmetry and Strain Induced Effects in Semiconductors (Wiley, New York, 1974).

[45] I. Vurgaftman, J. R. Meyer, and L. R. Ram-Mohan, "Band parameters for III-V compound semiconductors and their alloys," J. Appl. Phys. 89, 5815 (2001).

[46] A. Svizhenko and M. P. Anantram, "Effect of scattering and contacts on current and electrostatics in carbon nanotubes," Physical Review B 72, 085430 (2005).

[47] Y. Lee, M. Lannoo, N. Cavassilas, M. Luisier, and M. Bescond, "Efficient quantum modeling of inelastic interactions in nanodevices," Phys. Rev. B 93, 205411 (2016).

[48] U. Aeberhard and R. H. Morf, "Microscopic nonequilibrium theory of quantum well solar cells," Physical Review B 77, 125343 (2008).

[49] R. Lake, G. Klimeck, R.C. Bowen, and D. Jovanovic, "Single and multiband modeling of quantum electron transport through layered semiconductor devices," J. Appl. Phys. 81, 7845 (1997).

[50] A. Svizhenko, M. P. Anantram, T. R. Govindan, B. Biegel, and R. Venugopal, "Two-dimensional quantum mechanical modeling of nanotransistors," J. Appl. Phys. 91, 2343 (2002).

[51] N. Mori and T. Ando, "Electron-optical-phonon interaction in single and double heterostructures," Phys. Rev. B 40, 6175 (1989).

[52] E. B. Ramayya, D. Vasileska, S. M. Goodnick, and I. Knezevic, "Electron transport in silicon nanowires: The role of acoustic phonon confinement and surface roughness scattering," J. Appl. Phys. 104, 063711 (2008).

[53] S. Adachi, Properties of Group-IV, III-V and II-VI Semiconductors (John Wiley \& Sons Ltd, 2005). 